Perspectiva Geográfica

ISSN 0123-3769

Vol. 15/2010; pp. 225-238

\title{
Caracterización espacio-temporal y determinación de los patrones territoriales de alta concentración de la accidentalidad en la vía Tunja-Moniquirá
}

Space -time characterization and determination of territorial patterns of high accident concentration

of Tunja Moniquirá route

María del Pilar Camacho*

\section{Resumen}

La accidentalidad vial se ubica entre las primeras causas de mortalidad en todo el mundo; en Colombia se evalúa cada año el número de accidentes en las carreteras, y se obtienen cifras que realmente dicen muy poco, pues aunque en estos estudios se tienen en cuenta el lugar y la hora, no se analiza la relación de estos datos con las condiciones biofísicas predominantes. El objetivo de esta investigación fue caracterizar espacial y temporalmente los accidentes de tránsito ocurridos en la vía Tunja Moniquirá, y establecer los patrones territoriales de alta concentración, para proponer un programa preventivo. El aporte de este estudio radica en que se identifican características como la concentración de los accidentes por horas y lugares, y las particularidades biofísicas; se describe el diseño de la vía, y se relacionan todas las características por medio de un análisis estadístico, para identificar cuáles tienen más relevancia al momento de identificar las causas asociadas al accidente.

Palabras clave: Accidentalidad vial, Concentración de accidentes, Condiciones biofísicas, Patrón territorial.

Magíster en Geografía. Profesional Especializado-Coordinador SIG, Unidad Administrativa Especial de Parques Nacionales Naturales de Colombia Territorial Orinoquia.marpilca@gmail.com 


\begin{abstract}
Road accidents have been found to be among the primary causes of mortality throughout the world. In Colombia every year the number of accidents on the roads is assessed, but these figures really tell very little, since even though, these studies take into account the place and time of the accidents, they fail to take into account the relationship between the prevailing biophysical conditions at each location and the data collected. The objective of this research was to characterize both spatially and temporally the traffic accidents occurred in the Tunja $\sim$ Moniquirá route, and to establish high concentration territorial patterns in order to propose a preventive program. The contribution of this study lies in the identification of characteristics such as the concentration of accidents for hours and locations, and their specific biophysical particularities; it also describes the design of the roads, and relates all the characteristics through a statistical analysis to identify which have more relevance when identifying the causes associated with the accident.
\end{abstract}

Key words: Accident Concentration, Biophysical conditions, Territorial pattern, Traffic accidents.

226 María del Pilar Camacho 


\section{Introducción}

Según estudios de la Organización Mundial de la Salud (2005), las defunciones por lesiones causadas por el tráfico vehicular (LTV), y en particular por accidentes de tránsito de vehículos de motor (ATV), representan en todo el mundo una epidemia silenciosa que afecta a todos los sectores de la sociedad: "Las muertes relacionadas con el tránsito entre los años 1997 y 2000 fue la décima entre las principales causas de defunción en la población en general, se estima que han muerto 1,26 millones de personas en todo el mundo por causas relacionadas con LTV y el $90 \%$ vivían en países de bajo o mediano ingreso". En América Latina, según estos mismos estudios, la accidentalidad vial es la segunda causa de muerte.

Las cifras nacionales presentan la misma tendencia, ya que "en el período comprendido entre 1995 y 1999 las muertes violentas relacionadas con accidentalidad vial en el país alcanzaron cerca del $20 \%$ del total de las muertes registradas a nivel nacional (37.549 muertes en accidentes de tránsito)" (Rodríguez et al., 1999). Según el Instituto Nacional de Vías, en el periodo 1986-2006 el número de accidentes en el país pasó de 64.289 a 186.362 , dato que podría no ser muy diciente, si se tiene en cuenta que el número de vehículos también ha aumentado en un poco más de tres millones, pero el diseño, la velocidad, la capacidad de carga y las condiciones de protección para el conductor también han evolucionado, lo cual haría pensar que se han hecho más seguros, quedando entonces el interrogante de por qué los accidentes han aumentado en un poco más de 122.000 , pese a la mencionada evolución en las características de las vías y los vehículos.

La relevancia de la geografía en este estudio se manifiesta en la posibilidad de hacer un análisis que relacione de manera sistemática el número, el tipo, el lugar y las consecuencias del ATV, permitiendo una visión más amplia del fenómeno y la identificación de patrones territoriales de ocurrencia. Al respecto, afirma Harvey, "la geografía trata de la descripción y explicación de áreas diferenciadas en la superficie terrestre" (1969: 27). En este marco, el proyecto genera como aporte la interpretación espacial del fenómeno de accidentalidad. Con base en tal interpretación se busca encontrar los factores que determinan la peligrosidad de las vías analizadas y la relación entre estas y los factores reales que inciden en los accidentes.

\section{Planteamiento del problema}

El problema de investigación que se plantea es: ¿Es posible realizar una caracterización espacial y temporal de los accidentes de tránsito ocurridos en la carretera que conecta la ciudad de Tunja con Moniquirá, estableciendo los patrones territoriales de alta concentración, para proponer un programa preventivo?

La vía objeto de estudio se encuentra ubicada en la zona centro del departamento de Boyacá, y conecta los municipios de Moniquirá, Arcabuco y 
Motavita con Tunja, la capital del departamento; además, es corredor para acceder al norte del país, ya que por esta vía se llega a Bucaramanga, Cúcuta y la costa atlántica. El relieve predominante es el montañoso y las temperaturas, según estudios de suelos del Instituto Geográfico Agustín Codazzi, varían entre los 3 y los 17 grados Celsius.

\section{Metodología}

\subsection{Determinación de la intensidad de uso y de la variación en los volúmenes de tránsito de la vía}

La determinación del volumen de tránsito busca tipificar la vía, estableciendo su patrón de uso e identificando el vehículo que más la transita y los días de la semana que mayor volumen presentan, para con ello identificar la relación entre alta concentración diaria, horaria y su relación con el tipo de vehículo.

La situación actual de las variaciones en el tránsito promedio de esta vía se determinó de acuerdo con los conteos manuales que realiza en todo el país el Instituto Nacional de Vías (INVIAS); como resultado se obtiene la tabla que se presenta a continuación, la cual resume por estaciones el volumen de vehículos. La información en la columna TPDS (Tránsito Promedio Diario Semanal) muestra el volumen promedio de vehículos, y los números en las casillas indican el porcentaje de representa para vehículos pequeños, buses y camiones; en la casilla DESV se muestra la deviación estandar de los datos; la columna Total resume la información de camiones, y las casillas siguientes muestran el dato para cada tipo de vehículo y el porcentaje de representatividad de cada uno.

Tabla. Tránsito promedio diario estaciones 95, 96, 97 y 98 para el año 2007

\begin{tabular}{|c|c|c|c|c|c|c|c|c|c|c|c|c|}
\hline $\begin{array}{l}\text { ESTA- } \\
\text { CIÓN } \\
\text { No. }\end{array}$ & SECTOR & $\begin{array}{c}\text { LONG } \\
\mathrm{km}\end{array}$ & & PDS & & DESV & TOTAL & $C-2 P$ & $\mathrm{C}^{-} 2 \mathrm{G}$ & $\begin{array}{c}C-3- \\
4\end{array}$ & C - 5 & $>\mathrm{C}-5$ \\
\hline \multirow{2}{*}{95} & \multirow{2}{*}{ SOTE-TUNJA } & \multirow{2}{*}{14} & \multicolumn{3}{|c|}{2.287} & \multirow{2}{*}{156} & \multirow{2}{*}{4.280} & 1.142 & 2.035 & 387 & 124 & 592 \\
\hline & & & 61 & 12 & 27 & & & $26,68 \%$ & $47,55 \%$ & $9,04 \%$ & $2,90 \%$ & $13,83 \%$ \\
\hline \multirow{2}{*}{96} & \multirow{2}{*}{ ARCABUCO- SOTE } & \multirow{2}{*}{29} & \multicolumn{3}{|c|}{2.414} & \multirow{2}{*}{183} & \multirow{2}{*}{5.850} & 1.229 & 2.468 & 502 & 221 & 1.430 \\
\hline & & & 56 & 10 & 35 & & & $21,01 \%$ & $42,19 \%$ & $8,58 \%$ & $3,78 \%$ & $24,44 \%$ \\
\hline \multirow[t]{2}{*}{97} & \multirow{2}{*}{$\begin{array}{l}\text { MONIQUIRÁ- } \\
\text { ARCABUCO }\end{array}$} & \multirow{2}{*}{24} & \multicolumn{3}{|c|}{2.044} & \multirow{2}{*}{216} & \multirow{2}{*}{5.107} & 1.232 & 2.007 & 327 & 204 & 1.337 \\
\hline & & & 53 & 11 & 36 & & & $24,12 \%$ & $39,30 \%$ & $6,40 \%$ & $3,99 \%$ & $26,18 \%$ \\
\hline \multirow[t]{2}{*}{98} & \multirow{2}{*}{$\begin{array}{l}\text { BARBOSA- } \\
\text { MONIQUIRÁ }\end{array}$} & \multirow{2}{*}{9} & 3.77 & & & \multirow{2}{*}{166} & \multirow{2}{*}{6.477} & 1.833 & 2.693 & 355 & 155 & 1.441 \\
\hline & & & 67 & 8 & 24 & & & $28,30 \%$ & $41,58 \%$ & $5,48 \%$ & $2,39 \%$ & $22,25 \%$ \\
\hline
\end{tabular}

Fuente: Instituto Nacional de Vías INVIAS.

Desv: Desviación estándar

C - 2P: Camión de dos ejes pequeño

C - 2G: Camión de dos ejes grande
C - 3- 4: Camión y tracto camión de tres y cuatro ejes

C-5: Tracto-camión de cinco ejes

$>C-5$ : Tracto-camión de más de cinco ejes

228 María del Pilar Camacho 
3.2 Ubicación espacial y temporal de los accidentes de tránsito y determinación de patrones territoriales de concentración del fenómeno

Con la ubicación del lugar de ocurrencia de cada accidente y la identificación de las horas del día en las cuales se presenta el fenómeno, se pretende establecer patrones para identificar las características asociadas al relieve, al clima y a las condiciones del diseño vial, entre otras.

La información de los accidentes fue obtenida de dos fuentes: el Programa de investigación en tránsito y transporte de la Universidad Nacional de Colombia, el cual aportó las coordenadas GPS para accidentes ocurridos entre el años 2004 y 2007, y los informes de accidentalidad de los años 2002 al 2005 del INVIAS. Con esta información se procedió a establecer los lugares de alta concentración del fenómeno y a identificar patrones de ocurrencia de este. La información que se recolectó del INVIAS consistió en 121 puntos distribuidos entre los años 2002, 2003, 2004 y 2005; esta información se encuentra localizada por puntos de referencia, que se ubican dentro del mapa con el kilómetro de ocurrencia, y relaciona, además, la fecha, la hora, el número de heridos y el número de muertos. La información que se consiguió a través del Programa de investigación en tránsito y transporte está relacionada con un proyecto de investigación en el que están georeferenciandos todos los puntos de accidentes a nivel nacional, tabulando la información que recoge la policía de carreteras y asociándola a los puntos. Para la vía objeto de estudio se encontraron 42 puntos, ubicados entre Tunja y Barbosa, estando solo uno fuera del perímetro del municipio de Moniquirá, razón por la cual se toman en cuenta todos ellos. En el mapa anexo

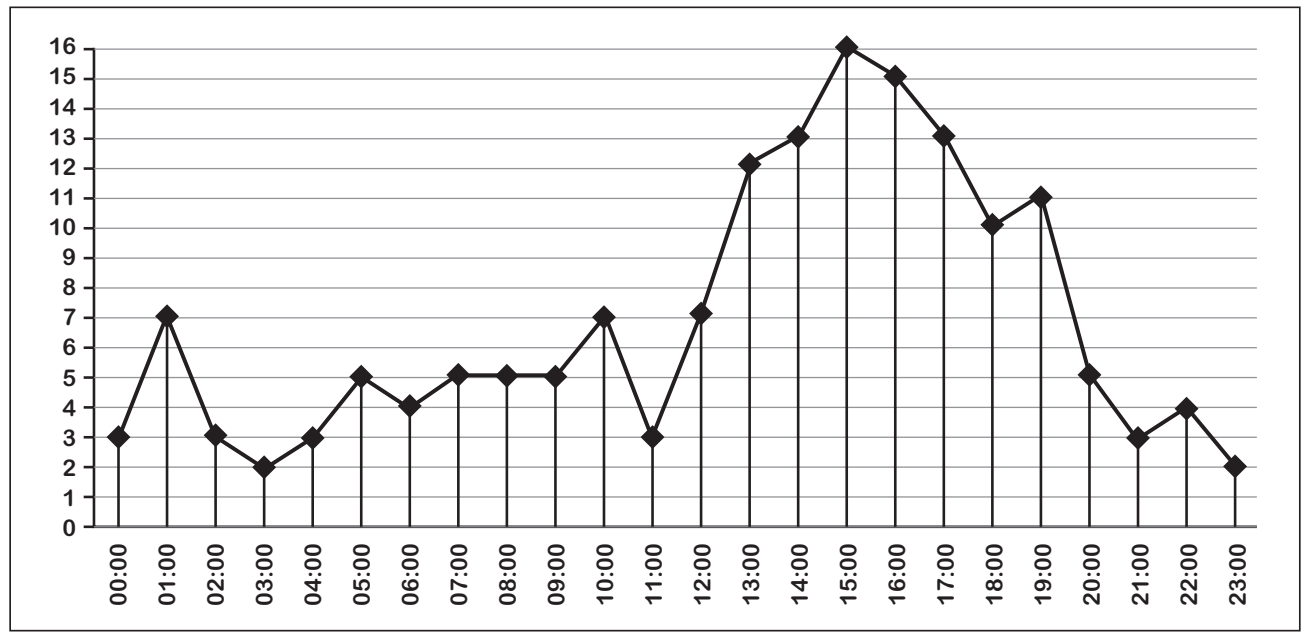

Figura 1. Concentración de los accidentes por horas

Fuente: Elaboración propia a partir de información obtenida por el Programa de investigación en tránsito y transporte de la Universidad Nacional de Colombia. 
se puede ver la ubicación espacial de los accidentes, el tramo de la vía en el que sucedieron y el municipio al cual pertenecen y en las figuras que se presenta a continuación se relacionan cada uno de ellos con sus características más relevantes, es decir, fecha de ocurrencia, día de la semana, hora y ubicación.

La Figura 1 muestra que el lapso con mayor representatividad está entre las 15:00 y las 16:00 horas, con 16 accidentes; además, el mayor número de accidentes se presenta luego de las 12:00, a excepción de los accidentes ocurridos a las 10:00 y a la 1:00, mostrando otro dato atípico.

\subsection{Identificación de puntos críticos en la vía}

La relación entre la hora de ocurrencia de los accidentes y el volumen de vehículos que pasan en esa hora por la vía sirve para determinar el índice de accidentalidad por cada mil vehículos.

Como se puede ver, el índice muestra que la hora en que el volumen de accidentes con respecto al volumen de vehículos es más alto corresponde a la 1:00, seguida de las 23:00. La concentración está muy cercana, lo cual hace pensar que hay algún factor externo que sirve de detonante o factor contribuyente a la ocurrencia del fenómeno. Se puede observar, además, que el índice de accidentalidad por cada mil vehículos entre las 15:00 y las 19:00 horas muestra concentraciones similares; sin embargo, no habría una relación evidente entre las horas de ocurrencia, pues las condiciones de brillo solar son diferentes en estas dos horas, y el volumen de vehículos desciende sustancialmente.

\subsection{Descripción del diseño de la red vial y determinación de las condiciones actuales de seguridad vial y velocidad}

Con el objeto de afrontar la calificación del cumplimiento de las condiciones de la red vial, comenzaremos por estructurar las que para los expertos son las condiciones que actualmente debe cumplir una vía; para ello nos permitimos citar a Antonio Chocontá, quien determina que

El diseño de una carretera debe hacerse de tal forma que el conductor de un vehículo no deba tomar sino una decisión cada vez y que nunca se vea sorprendido por situaciones inesperadas en las que deba tomar decisiones sin que tenga suficiente tiempo para reaccionar. Los accidentes ocurren más frecuentemente donde y cuando se presentan varias situaciones ante las cuales debe reaccionar el conductor simultáneamente (1998: 54).

También indica que "las carreteras se diseñan para proporcionar viajes seguros, eficientes y cómodos", y agrega que, para lograrlo, el diseño de estas debe contemplar las mejores técnicas de la ingeniería, de manera que se presenten la menor cantidad de accidentes; ya que "los accidentes rara vez son producidos por causas sencillas; por el contrario, varias circunstancias afectan generalmente la situación para que se

230 María del Pilar Camacho 


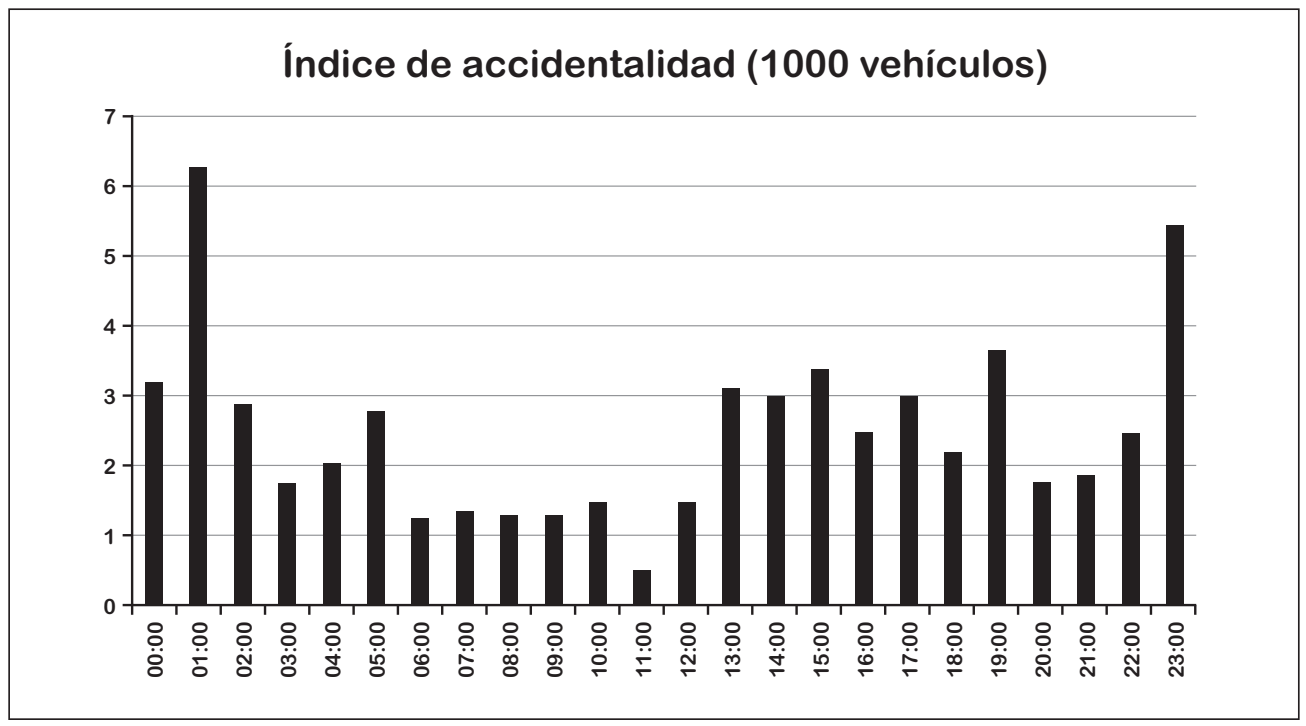

Figura 2. Índice de accidentalidad

Fuente: Elaboración propia a partir de información obtenida por el Programa de investigación en tránsito y transporte de la Universidad Nacional de Colombia y el Instituto Nacional de Vías, INVIAS.

genere un accidente" (Chocontá, 1998: 53). Argumenta también que estas circunstancias pueden verse intervenidas por los tres elementos que concurren en la operación: la vía, el vehículo y el conductor; sin embargo, pone de manifiesto que hay que tener presentes otros factores que fácilmente pueden desencadenar un accidente.

En la vía se observaron dos velocidades directrices: en la zona entre Barbosa y Arcabuco, de 30 y $40 \mathrm{~km} / \mathrm{h}$, y entre Arcabuco y Tunja, de $60 \mathrm{~km} / \mathrm{h}$. Estas velocidades fueron establecidas desde 1950, con el proyecto BIRF de rediseño de la vía; desde entonces no se han hecho mayores ajustes a la velocidad ni al trazado, simplemente se han hecho arreglos al afirmado y ampliaciones que eliminaron las cunetas y las bermas.
De acuerdo con las características identificadas de la vía, y teniendo en cuenta que el volumen de vehículos es bajo, se puede determinar que las velocidades de operación están adaptadas a las características de diseño de la vía vigentes desde hace 50 años, razón por la cual es de suponerse que no están acorde con las actuales, consignadas en el manual de Especificaciones y Normas de construcción de carreteras INVIAS-2007.

\subsection{Espacialización de las condiciones biofísicas predominantes en la vía}

La relación de las condiciones biofísicas con el fenómeno de la accidentalidad es directa, ya que las condiciones climáticas pueden desencadenar situaciones adversas para el conductor, como pérdida de visibilidad, 
derrumbes, inundaciones en la vía y pérdida de fricción de las llantas al pavimento, entre otras; de manera que conocer las condiciones predominantes en la zona permitirá hacer un análisis acerca del tipo de influencia que ejerce cada uno de los fenómenos en la ocurrencia de los accidentes.

Para tener una visión más objetiva de los posibles factores detonantes del accidente es necesario conocer las condiciones biofísicas de la zona objeto de estudio. Al consultar literatura sobre el tema se encuentra información escrita y geográfica producida por distintas entidades, como el Instituto Geográfico Agustín Codazzi (IGAC), la Corporación Autónoma Regional de Boyacá (Corpoboyacá) y el Instituto de Hidrología, Meteorología y Estudios Ambientales de Colombia (IDEAM), que permite hacer un análisis de cuáles son las condiciones biofísicas predominantes en la zona.

El régimen de lluvias consolidado para el año se comporta, como lo describe el estudio de suelos del IGAC, con una precipitación anual de 700 milímetros en los meses de "verano" y hasta 4000 milímetros en los meses de "invierno". Además, las curvas de precipitación muestran una concentración de lluvia en la zona más baja, aledaña a Moniquirá, y una precipitación media en la zona más alta, aledaña a Tunja. El mes en el que más precipitación se presenta es octubre, y el con menos precipitación es enero. Las condiciones atmosféricas se convierten en riesgo en la medida que los factores detonantes tengan mayor presencia en la vía.

Las formaciones geológicas encontradas en la zona de estudio pertenecen al cretáceo; son rocas sedimentarias de origen aluvial; a pesar de ser arcillolitas, el material es relativamente estable, pues no hay evidencias de afectaciones significativas por presencia de fallas ni deslizamientos.

3.6 Determinación de los factores de riesgo asociados a los fenómenos biofísicos que inciden en la accidentalidad, y utilizando análisis estadístico estimar el grado de incidencia de cada uno de ellos en la ocurrencia del fenómeno

El concepto de riesgo ha tenido hasta la fecha diversas interpretaciones. En términos generales, existe riesgo en cualquier situación en la que no se conoce con exactitud lo que ocurrirá en el futuro. Los factores de riesgo asociados a los accidentes fueron identificados en la visita de campo, tomando como base la información determinada por el Programa de investigación en tránsito y transporte de la Universidad Nacional de Colombia (PITT). Debido a que la falta de información es el factor identificado por el PITT que más se repite y que de acuerdo con la clasificación de factores asociados al accidente no tienen en cuenta las variables temperatura y humedad, y estas fueron las causas que las personas que transitan la vía identificaron como las principales causas de accidentalidad en determinados puntos, para este estudio se 
tomaron los valores de precipitación mensual y temperatura media como los factores de riesgo. Además de estos factores se tomaron el estado de conservación de la infraestructura vial (presencia de deslizamientos producidos por falta de mantenimiento, caída de árboles, fallas en el afirmado), el correcto diseño de las curvas (peraltes), la presencia de animales en la vía y algunas otras malas prácticas en los manejos de desechos de explotaciones de grava en el corredor vial.

\subsection{Análisis estadístico para estimar el grado de incidencia de cada uno de los factores en la ocurrencia del fenómeno}

Es necesario hacer un análisis de la incidencia de los factores de riesgo y de los factores detonantes ${ }^{1}$; por esto se usan técnicas estadísticas que no solo permiten la estimación de cada una de estas variables, sino que aportan medidas para establecer la calidad del modelo usado y el sesgo implicado en la inclusión de cada una de las variables. Los factores de riesgo y los factores detonantes (variables independientes para el modelo estadístico) identificados en la vía son los siguientes:

- Precipitación mensual

- Temperatura media

- Presencia de deslizamientos producidos por falta de mantenimiento

- Caída de árboles

- Fallas en el afirmado
- Correcto diseño de las curvas

- Presencia de animales en la vía

- Mal drenaje en la vía

Con estas variables se realizó una primera estimación, usando un modelo lineal de la forma $f(\mu)=X \beta$, para determinar el grado de incidencia de cada una de estas variables en la ocurrencia del fenómeno (determinada por el número de accidentes). El resultado implicó la reducción del número de variables independientes a las que se citan a continuación:

Precipitación: Volumen promedio de lluvia al año

Temperatura: Grados Celsius predominantes en la zona

Peralte: Identifica si en el sitio del accidente se presentan fallas en el peralte de la vía

Drenaje: Identifica si en la vía se presentan fallas en el drenaje

Señalización: Identifica las zonas en las cuales la señalización es mala

Los datos se tabularon nuevamente, identificando para cada kilómetro el número de accidentes (variable independiente) y el valor medido para cada una de las variables citadas.

1 Eventos que no se tienen presentes cuando se va conduciendo (por ejemplo, huecos, alcantarillas sin tapa, rizados en la vía, animales, etc.), que generan reacciones abruptas, como frenadas en seco o cambios bruscos en la dirección (Sáenz, Néstor, Apuntes de clase). 
3.8 Hacia un programa preventivo basado en la autorregulación, más que en la prohibición

Los accidentes de tránsito son una importante causa de mortalidad, morbilidad e invalidez en nuestro país. Es importante determinar por qué se produce un accidente, dado que para explicarlo se requiere de una combinación de factores en la que intervienen, entrelazadas con los hechos mecánicos y aquellos del entorno, las decisiones tomadas por los conductores. Debe admitirse que todo vehículo en circulación es conducido por una persona que no es un ingeniero mecánico y que adopta decisiones por su cuenta; lo que circula es un sistema conductor/vehículo que se desplaza bajo el dominio de su conductor, quien mide, juzga y decide acciones, respondiendo a las señales externas que recibe, dentro de los límites de su natural capacidad de evaluar datos y tomar decisiones. La determinación de las causas permite esbozar el porqué, identificando los factores asociados a cada accidente, permitiendo establecer patrones, fallas en el sistema y soluciones prácticas que se pueden aplicar para prevenir los accidentes.

En esas condiciones se introduce el análisis de la determinación de valores subjetivos, tales como la autorregulación, que se define como el "proceso voluntario de control y monitoreo interno que tiene una organización con capacidad para el análisis real de las situaciones, con fines de hacer una autocrítica para llevar a cabo, y de manera flexible, los correctivos de lugar"
(Andón, 2005: 1). Puede decirse entonces que es ajustarse de manera voluntaria a las normas y leyes de una sociedad.

De acuerdo con la orientación que se pretende dar a la propuesta de plan de prevención de autorregulación, se presentan los objetivos y las metas a corto, mediano y largo plazo, que se proponen para reducir el número de accidentes en la vía objeto de estudio.

\section{Objetivos}

\section{General}

Disminuir los factores de riesgo potencial presentes en la vía.

\section{Específicos}

Identificar, con ayuda de estadísticas en los puntos críticos de accidentalidad, cuáles son las causas asociadas que más influyen en la ocurrencia del accidente.

Implementar auditorias de seguridad vial periódicamente.

Generar conciencia en los conductores, los peatones y las autoridades, de manera que las lesiones asociadas a accidentes de tránsito se puedan prevenir.

Metas

\section{1) A corto plazo}

\section{Aspectos preventivos}

La eficacia en la actuación sobre los factores de riesgo ambientales es 
variable; tratar de controlar la velocidad durante una neblina o una lluvia es una responsabilidad de cada conductor. Es recomendable el adecuado estudio de los factores que causan los accidentes de tránsito; esto se debe realizar con la participación de toda la ciudadanía.

La eficacia en la seguridad del vehículo; esto implica la revisión diaria del automóvil, tomando en cuenta los frenos, las llantas, el aceite, el combustible, las correas de seguridad, la silla para los bebés. Está demostrado que con la aplicación de medidas preventivas nos evitamos muchos accidentes.

La identificación de los factores de riesgo asociados al conductor, quien debe conocer los problemas que se pueden presentar con la presencia de factores detonantes.

La construcción de buenas carreteras, amplias, bien señalizadas, de acuerdo con las características de diseño determinadas por las entidades reguladoras.

\section{2) A mediano plazo}

\section{Aspectos correctivos}

Esta meta está orientada al cambio en las prácticas diarias que desarrollan los conductores.

Buena parte de los accidentes de tránsito podrían evitarse si los conductores adoptaran las consignas del manejo defensivo, que se basa en la premisa de que no importa qué hagan los otros autos, la seguridad propia depende de uno mismo.

Mantener dos segundos de distancia con el vehículo de adelante, cuando están en movimiento; con los vehículos detenidos es necesario mantenerse a una distancia que nos permita ver el contacto de las ruedas del carro vecino con el suelo y así evitar choques en cadena o colisiones si el otro conductor arranca su vehículo por error.

El consumo de alcohol, drogas y sedantes disminuye la capacidad de reacción, los reflejos y la visión. Una copa de vino o una botella de cerveza tardan una hora en eliminarse del organismo. Los sedantes tienen efecto residual, por ello, si se consumen por la noche lo mejor será no conducir en la mañana siguiente.

\section{3) A largo plazo}

\section{Aspectos autorreguladores}

La toma de conciencia por todos los actores involucrados en la aplicación de las normas es un proceso que toma tiempo, pero sus resultados son arrolladores, pues si se crea la cultura de la no violación de las normas, sin importar si hay condiciones favorables para violarlas, es decir, el absoluto conocimiento de la norma y su aplicación sin excusas, se reducirá la ocurrencia de fenómenos como el de la accidentalidad. 


\section{Resultados}

Al comparar las estaciones y las diferentes fechas se observa que el volumen de vehículos que transitan esta vía se concentra entre las 9:00 y las 19:00, con un pico entre las 17:00 y las 19:00; que el volumen de vehículos es representativo en los días miércoles, jueves y viernes, y que el tipo vehículo que más transita por esta vía es el pequeño, con un porcentaje promedio de $50 \%$; teniendo en cuenta que hay 6 categorías más de vehículos, se puede deducir que la representatividad de los camiones grandes y los buses no es muy alta. También se evidencia que durante los años contados, los volúmenes de tránsito, por horas y días de la semana, muestran cómo el número de vehículos va de menos a más en la medida en que avanza el día, sin importar cuál día de la semana sea; de tal manera que se esperaría que la ocurrencia más alta de accidentes se presente entre el lunes y el jueves. Las horas pico representan datos atípicos, que no son muy representativos, pues no siguen ningún patrón; sin embargo, se tendrán en cuenta para analizar la ocurrencia del fenómeno de la accidentalidad. Se puede identificar que el tránsito promedio diario registrado en las estaciones no muestra concentraciones significativas en ningún día en particular.

Los accidentes se distribuyen a lo largo de la vía de manera uniforme, aunque se pueden identificar tres puntos críticos, en los cuales se concentran más accidentes: el kilómetro 37, el kilómetro 51 y entre el kilómetro 45 y el 48; sin embargo, no se registran patrones de concentración en cuanto el día y la hora de ocurrencia en alguno de estos puntos.

Los volúmenes de tránsito promedio diario (TPD) presentan alta concentración entre las 9:00 y las 19:00, y baja concentración entre las 00:00 y las 9:00, y las 19:00 y las 23:00. La distribución temporal de los accidentes no presenta patrones homogéneos. El día con mayor representatividad es el sábado, y la hora con mayor representatividad se ubica entre las 15:00 y las 16:00.

La vía objeto de estudio data de 1950; con el devenir de los años cambió de función e importancia, pasó de vía terciaria a vía nacional pavimentada territorial, pero sus características de diseño no se modificaron y las transformaciones que ha tenido son básicamente ampliaciones y mejoras en el afirmado. Al tomar los patrones de referencia para las vías nacionales, de acuerdo con el Manual de Diseño de Carreteras, se concluye que la vía Nacional Pavimentada Territorial 6.209 no cumple con las condiciones mínimas de seguridad.

Luego de correr el modelo dos veces y obtener resultados, se concluye que las variables precipitación y temperatura no mejoran su significancia en la estimación del número de accidentes en ausencia de la variable dicótoma peralte, lo que refuerza la necesidad de obviarlas. Adicionalmente se realizó una gráfica del número de accidentes en función de la 
precipitación, la temperatura y el drenaje; esto permitió identificar que en los kilómetros 45 y 37, que presentan alta ocurrencia de accidentes, no se presentarían estimaciones altas de acuerdo con los parámetros estimados en este modelo; por esto se concluye que el primer modelo es el más indicado para estimar el número de accidentes por kilómetro, y que las variables peralte y drenaje son las más representativas.

\section{Conclusiones}

La concentración de vehículos muestra que no existe un patrón en la ocupación de la vía, lo que hace muy difícil establecer días de la semana con rangos diferentes, u horas del día con concentraciones que hagan pensar que pueden presentarse datos atípicos en la ocurrencia del fenómeno de la accidentalidad; sin embargo, solo en el momento de comparar la información del volumen con la de los accidentes se podrá establecer un patrón de ocurrencia.

El tránsito promedio diario registrado en las estaciones para los tres años es homogéneo, no muestra concentraciones significativas en algún día en particular.

El volumen consolidado total de vehículos es de 2.630 , de los cuales, el $60 \%$ son vehículos pequeños, el 10,25\% son buses y el $29,75 \%$ restante son camiones.

En la vía no se evidencia un mantenimiento preventivo, lo que pone al descubierto la ausencia de auditorías de seguridad vial, que deja como resultado el incumplimiento de las especificaciones y normas de seguridad vial.

Las formaciones geológicas encontradas en la zona de estudio pertenecen al cretáceo, son rocas sedimentarias de origen aluvial; a pesar de ser arcillolitas, el material es relativamente estable, pues no hay evidencias de afectaciones significativas por presencia de fallas ni deslizamientos, situaciones que hacen relativamente estable la vía en cuanto a deslizamientos, derrumbes o pérdidas en la bancada.

Cuando un vehículo transita por primera vez un sector lo hace con mayor nivel de seguridad, pues su conductor va con mayor precaución, aumenta sus niveles de concentración y, por lo tanto, disminuye la velocidad, evita distracciones y en el momento que aparecen condiciones biofísicas adversas aumenta las precauciones hasta cierto nivel. Sin embargo, si no existe la cultura de la autorregulación estas precauciones no sirven de nada, pues la constante violación de las normas hace que se presenten factores adversos que no es posible controlar. 


\section{Literatura citada}

Andón, A. (2005). Falacias de la Autorregulación, obtenido el 20 de mayo de 2010, desde http://alvaro-andon.blogspot.com/2005/06/falacias-de-la-autorregulacion.html

Chocontá R., Pedro A. (1998). Diseño geométrico de vías. Bogotá: Escuela Colombiana de Ingeniería.

Harvey, D. (1969). ExpIanation in Geography. London: Edward Arnold.

Ministerio de Transporte. (2007). Plan Nacional de Seguridad Vial 2004 - 2006. "Hacia una nueva cultura de seguridad vial”. Bogotá: Fondo de Prevención Vial.

Organización Mundial de la Salud, OMS. (2005). Defunciones por accidentes de tránsito en países seleccionados de las Américas, 1985-2001. México.

Rodríguez, D.; Fernández, F. J.; Acero V., H. (2003). "Road traffic injuries in Colombia”. Injury Control and Safety Promotion, Vol. 10, №1-2. 